\title{
Gradhiva
}

GRADHIV

Revue d'anthropologie et d'histoire des arts

$30 \mid 2019$

Précieux

\section{Chroniques d'un musée. Une folle décennie à la loupe}

\section{Éléonore Devevey}

\section{(2) OpenEdition}

\section{Journals}

Édition électronique

URL : http://journals.openedition.org/gradhiva/4734

DOI : 10.4000/gradhiva.4734

ISSN : 1760-849X

Éditeur

Musée du quai Branly Jacques Chirac

\section{Édition imprimée}

Date de publication : 4 décembre 2019

Pagination : 143-151

ISBN : 978-2-35744-113-2

ISSN : 0764-8928

\section{Référence électronique}

Éléonore Devevey, " Chroniques d'un musée. Une folle décennie à la loupe », Gradhiva [En ligne], 30 2019, mis en ligne le 31 mars 2021, consulté le 02 avril 2021. URL : http://journals.openedition.org/ gradhiva/4734; DOI : https://doi.org/10.4000/gradhiva.4734

(c) musée du quai Branly 
Chroniques d'un musée. Une folle décennie à la loupe.

Éléonore Devevey 


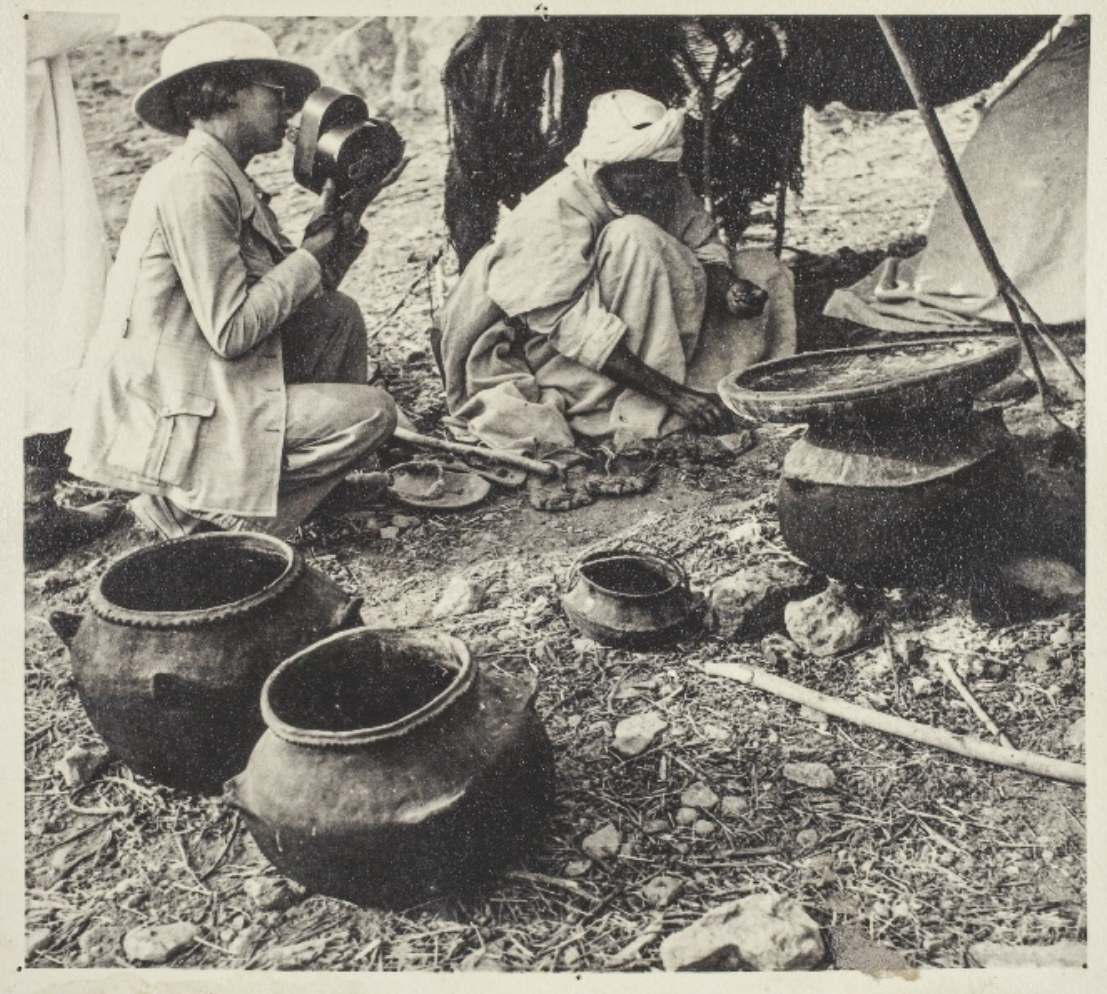


Note de lecture
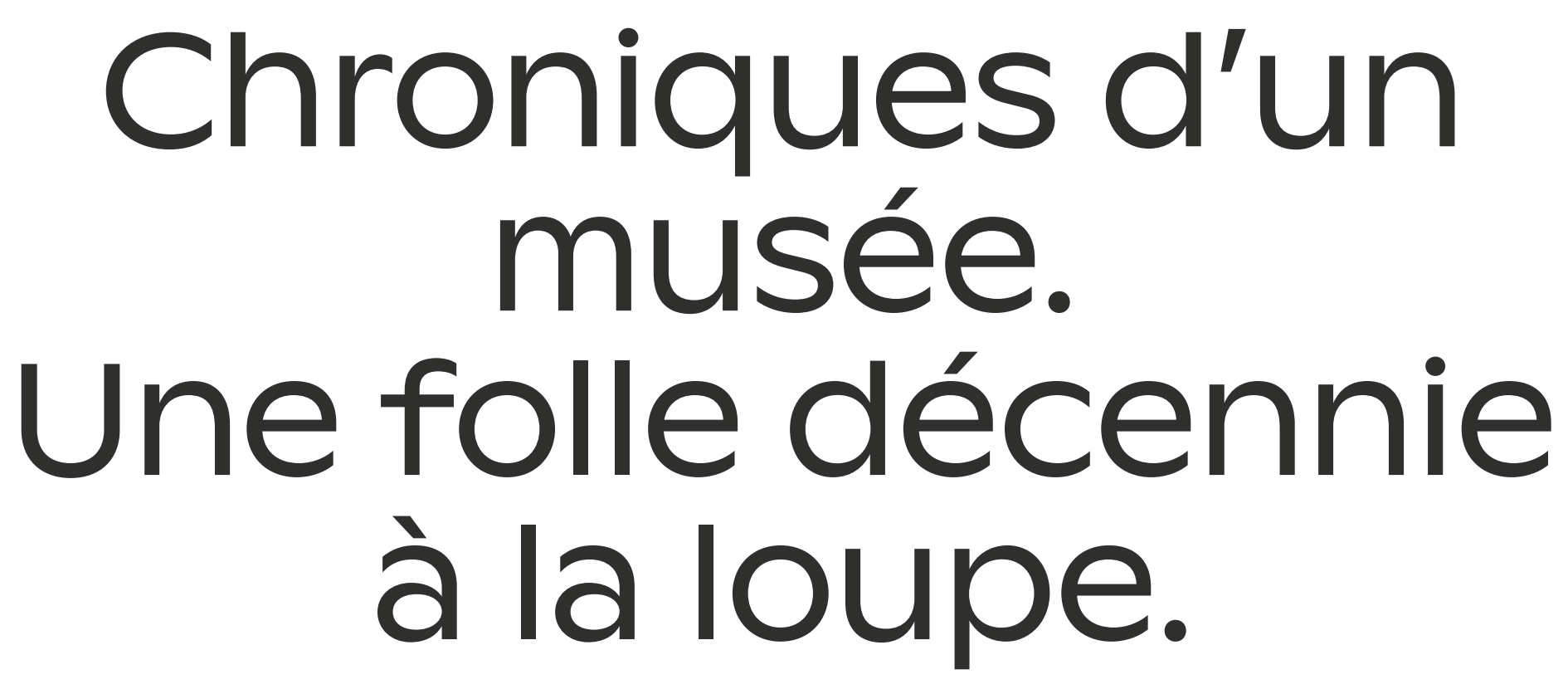

À propos d'André Delpuech, Christine Laurière

et Carine Peltier-Caroff (dir.), Les Années folles

de l'ethnographie: Trocadéro 28-37. Paris,

Publications scientifiques de MNHN, coll.

«Archives», 2017, 1007 p.

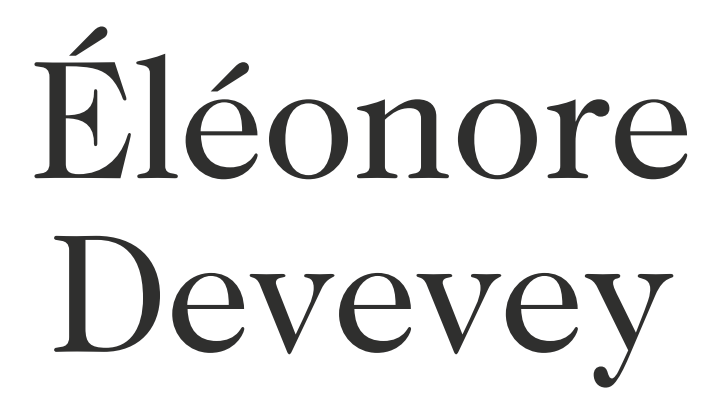




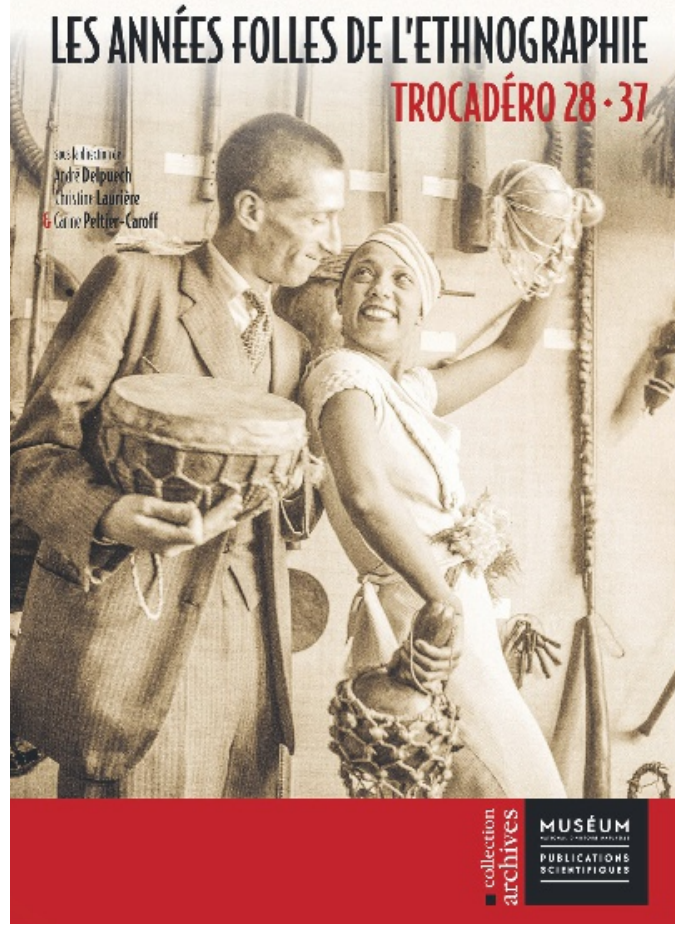

Boris Lipnitzki (1887-1971) : Georges Henri Rivière et Joséphine Baker à l'exposition de la mission Dakar-Djibouti, dans la galerie rénovée d'Afrique noire du musée d'Ethnographie du Trocadéro, juin 1933. Roger-Viollet/Lipnitzki.

L'histoire de l'ethnologie française dans I'entre-deux-guerres a, depuis une quinzain d'années, été arpentée suivant des biais multiples: la vie savante de ses grandes figures, sa mission phare (Dakar- Djibouti), ou encore ses démêlés avec la littérature. $\mathrm{Si}$ I'histoire des premières décennies du musée d'Ethnographie du Trocadéro (MET) et celle de son successeur, le musée de I'Homme, ont fait l'objet d'importants volumes ${ }^{1}$, la période charnière de «mue» entre le «vieux Troca » et l'ouverture du nouveau musée, en 1937, restait cependant à examiner en détail. Une lecture rétrospective trop rapide tend à faire du MET rénové le brouillon du musée de l'Homme à venir sa brillante préhistoire. Les Années folles de l'ethnographie, somme coordonnée par André Delpuech, Christine Laurière et Carine Peltier-Caroff, propose un état des lieux dynamique du vieux musée mis à neuf, et restitue, suivant un parti kaléidoscopique, la singularité et l'effervescence de sa dernière décennie d'existence. C'est assurément une décennie foisonnante, au cours de laquelle I'institution (fondée en 1878) sort de sa léthargie et adopte une muséologie moderne. Le volume vient en un sens déployer la formule de Christine Laurière dans un article de 2003 , qui voyait passer le musée «du magasin de bric-à-brac à la sécheresse de l'étiquette ${ }^{2}$ »; cette rationalisation de ses ambitions et de son mode de fonctionnement ne l'empêche toutefois pas de traverser des «années folles», animées par l'enthousiasme des (re)commencements. Désormais conçue comme un «musée laboratoire», l'institution connaît une phase de modernisation et de popularisation, devenant alors un lieu en vogue, à la fois populaire et mondain, élitiste et militant, lieu de science et musée des beaux-arts.

Comme l'expose l'introduction (C. Laurière), cette «mue» a été le fait de deux changements majeurs, survenus en 1928: I'institution est alors rattachée au Muséum national d'histoire naturelle et bénéficie «d'une exceptionnelle direction à deux têtes, Paul Rivet et Georges Henri Rivière (GHR), qui scellent au printemps 1928 une alliance inédite et explosive entre la science et la culture» (p. 10). La renaissance du musée tient pour beaucoup à ce «tandem» directorial, tout en différences et complémentarité, mais elle est aussi une «œuvre chorale» (p. 19), polarisant des acteurs d'horizons très divers. L'élan collectif va ainsi faire du «musée Grévin de l'exotisme » (selon l'expression de GHR, p. 20) un lieu rayonnant corollaire de l'entreprise coloniale, mais destiné à lutter contre les préjugés esthétiques et raciaux, et que ses directeurs veillent à organiser comme «une intelligente bijouterie» (p. 44): une machine à régler, depuis les montres du personnel jusqu'aux entrées des fiches descriptives attachées à chaque objet, en passant par l'approvisionnement en antimite et la tenue des bals antillais du samedi soir. Depuis le MET rajeuni, I'ethnologie apparaît à la fois comme un «petit monde » et un monde en réseau, où les frontières internes comme externes restent poreuses. C'est donc la transformation d'un musée marginal et déliquescent en plate-forme de la discipline, au cœur du dispositif culturel et scientifique, que met en avant l'ensemble du volume. Ce faisant, il offre aussi un éclairage oblique sur la France des années 1930: ce ballet orchestré par Paul Rivet et GHR fait office de révélateur et de catalyseur de transformations culturelles et politiques plus globales, illustrant notamment l'évolution de la place de l'empire colonial dans la société française. On retiendra icila grande richesse et la diversité elle-même «chorale» des contributions, s'attachant à des objets d'étude variés, et surtout le faste des documents d'archives et de l'iconographie qui les accompagne, de la série de photographies de Joséphine Baker posant devant les vitrines de l'exposition «DakarDjibouti » à celle d'une «femme eskimo préparant des intestins de phoque pour la fabrication d'imperméables », d'un duel touareg sur les terrasses du palais (où Andre Leroi-Gourhan, costumé, joue l'un des combattants) aux relevés d'art rupestre africain, des notes de terrain aux inventaires du musée, en passant par la reproduction d'une publicité pour «Insectol, I'Allié de la mission Paul Coze contre les maringouins ». Les «encadrés», signés pour l'essentiel par C. Peltier-Caroff, font la lumière sur un détail insolite, une innovation ou un dispositif technique remarquable; les documents d'archives reproduits en annexe (telle une sélection de cinq textes de GHR, représentatifs de la diversité des supports et des registres qu'il savait mobiliser) apportent de précieux compléments. On se contentera ici de proposer un parcours cursif dans l'ensemble du volume, en suivant son architecture. L'ensemble des contributions se répartit en trois volets, examinant, pour simplifier, ce qui se joue 1) dans les murs du musée, 2 ) hors ses murs, et 3 ) dans ses prolongements médiatiques.

Le premier volet, «Objets d'ethnographie objets d'art? Entre science, culture ef mécénat», examine les rapports du musée avec l'art et le monde de l'art, les partis muséographiques et les modes de gestion des collections impulsés par la nouvelle direction.

L'exposition «Arts anciens de l'Amérique », orchestrée par GHR et Alfred Métraux entre autres, amorce au printemps 1928 renaissance du musée, et permet de ma l'entregent de GHR (C. Faucourt). Servie par une muséographie aérée, attentive à la visibilité de chaque œuvre, l'exposition, qui a lieu au pavillon de Marsan, mais dont le MET est le principal prêteur d'objets, signe aussi l'entrée des productions précolombiennes dans le domaine des arts: c'est, aux yeux des critiques, «une seconde découverte de l'Amérique» (p. 70). Se fait ainsi jour la nouvelle ligne du musée, la volonté d'œuvrer «dans le double domaine de l'art et de la science» (p. 61), de valoriser l'intérêt plastique des objets, sans négliger l'expertise ethnographique. C'est à l'issue de ce succès que Rivet engage GHR à ses côtés, en juin 1928

La contribution de F. Grognet expose les enjeux de la réorganisation du Trocadéro, de 1928 à 1931, soit la transition du «capharnaüm » au musée modèle, de la «honte nationale» (p. 85) à la vitrine de la discipline, lieu d'expérimentation muséographique et de recherche scientifique. Cette transition engage une «redéfinition de l'utilité sociale » (p. 79) de l'institution, qui se veut désormais lieu d'innovation pédagogique et corollaire de l'ambition coloniale. Passant par la rationalisation du processus constitutif des collections, cette dynamique implique à la fois la professionnalisation de l'ethnographie et la démocratisation du savoir qu'elle constitue elle a aussi pour effet l'institutionnalisation de l'«art primitif», supplantant l'«art nègre». Cette phase s'achève avec l'inauguration de la salle du Trésor, en 1932, dont le but est de mettre en valeur, suivant un parti pris résolument esthétique, une sélection de pièces exceptionnelles, qui deviennent par là même, «chefs-d'œuvre».
Voir Nélia Dias, Le Musée d'ethnographie du Trocadéro (1878-1908): anthropologie et muséologie en France,

Paris, Éditions du CNRS, 1991 ;

Alice Conklin, Exposer l'humanité

race, ethnologie et empire en France (1850-1950),

trad. par Agathe Larcher-Goseha, Paris, Muséum national d'histoire naturelle,

2015; Claude Blanckaert (dir.), Le Musée de l'Homme:

histoire d'un musée laboratoire,

Paris, Muséum National d'histoire naturelle, 2015.

2.

Christine Laurière, «Georges Henri Rivière au Trocadéro. Du magasin de bric-à-brac

à la sécheresse de l'étiquette", Gradhiva 33, 2003: 57-66. 
D'après la formule de Vincent Debaene, " "Étudier des états de conscience". La réinvention du terrain par l'ethnologie, 1925-1939», L'Homme 179, 2006: 18.
C. Laurière reconstitue I'histoire de la Société des amis du musée d'Ethnographie du Trocadéro (Samet), principal soutien «mora et financier » de la restructuration du musée. Conçue dès 1914, véritablement active à partir de 1928, la Samet est alors présidée par Charles de Noailles, atout mondain rallie par GHR, protecteur, avec son épouse Marie-Laure, de toutes les avant-gardes du moment, et entouré d'un petit groupe de mécènes actifs, figures du monde de l'art primitif (Paul Guillaume, Charles Ratton, Georges Wildenstein, David David-Weill). Les ressources ainsi rassemblées, suppléant celles, maigres, octroyées par les pouvoirs publics, seront d'abord utilisées pour le simple fonctionnement et l'entretien du musée (et notamment la contribution aux appointements du personnel), puis à des fins plus prestigieuses: acquisition d'objets, contribution aux missions et aux exposition temporaires, soirées à thème inventives et fastueuses, autant d'initiatives qui contribuen à faire rayonner l'ethnologie dans la cité.

Envisageant le Trocadéro au «miroir allemand », la contribution $\mathrm{d}^{\prime} \mathrm{H}$. Ivanoff éclaire l'intérêt des deux dirigeants du musée pour les institutions ethnologiques allemandes, et notamment pour l'Institut de morphologie culturelle de Leo Frobenius. C'est une exposition de peintures rupestres d'Afrique du Sud qui noue en 1930 les liens entre les deux institutions, et entre GHR et Frobenius, introduit à Paris par l'abbé Breuil. Le propos est d'abord de faire une place à la préhistoire «exotique» au sein du musée, et de révéler aux Parisiens l'Afrique comme «un fabuleux livre d'images en pierre » (p. 226). Mais l'enjeu d'une telle exposition n'est pas seulement scientifique et esthétique. Par comparaison avec le modèle allemand, l'ethnologie française a, dans les termes de Mauss, été en France "traitée en Cendrillon» (p. 223). Le MET doit rattraper son retard, en adoptant des partis analogues à ceux du musée de Berlin, restauré antérieurement et en se mettant au diapason international. Derrière la coopération institutionnelle, I'enjeu politique et colonial est bien présent les directeurs du MET savent faire jouer à bon escient les rivalités entre puissances européennes, tout en s'efforçant de contribuer à les pacifier.

«Un chantier des collections, un musée en chantier » (A. Delpuech, L. Mész, F. Servain-Riviale) restitue l'esprit et le déroulé du chantier de rénovation du musée et de rationalisation de la gestion des collections; celui-ci passe notamment par le rassemblement au musée de collection éparses entre les différentes institutions nationales et par une politique d'acquisition très dynamique - près de cinquante mille objets, issus de dons et de collectes, entrent au musée entre 1928 et 1935 . Quelques innovations marquent également les pratiques d'enregistrement, de catalogage e de conservation : I'enregistrement sous $\mathrm{X}$ des objets ayant perdu leur identification d'origine, la systématisation de la fiche descriptive, la création d'un laboratoire dédié à la préservation des objets. Ces «années folles » constituent ainsi «une étape décisive tant dans l'apport exceptionnel de collections que sur un plan méthodologique avec la mise en place d'outils muséaux modernes et novateurs, appelés à se perpétuer tout au long du $x x^{\mathrm{e}}$ siècle » (p. 236).

A. Martin se livre enfin à une minutieuse étud des «questions(s) d'étiquette(s) », à partir de l'examen systématique des inventaires et des procédés d'enregistrement et de catalogage, afin de «restitue $[r]$ le cheminemen et le résultat du travail d'immatriculation [...] des collections accomplis durant la décennie» (p. 285). A posteriori, la réalisation de ce travail, dirigé par Marcelle Bouteiller, se révèle parfois tâtonnante, voire déficiente, faute d'un personnel stable et qualifié. Le tableau recensant les étiquettes et les marquages présents sur les collections conservées au MET avant 1937, réalisé par A. Martin, vise à permettre aux chercheurs d'aujourd'hui qui travaillent sur ces objets de suivre plus fidèlement «l'histoire de leurs appropriations et pérégrinations successives » (p. 336).

La seconde partie du volume, «Un musée en réseau. Missions et collections ethnographiques », restitue l'esprit et I'ambition des missions ethnographiques qui achalandent le musée, portées par un «impérialisme colonial comme scientifique (p. 339), en examinant leurs temps forts et quelques cas peu connus.

Elle s'ouvre sur la figure de l'incontournable Marcel Mauss, plus exactement sur une étude consacrée à «sa manière d'habiter le rôle de professeur » (p. 347) à l'Institut d'ethnologie, dont il est le principal animateur - «the whole show», en ses propres termes (T. Hirsch). Le propos est ici de «tenter de saisir [I]a parole, ses ressorts et ses effets » (p. 352) de ce «maître sans livre » (p. 348), dont les cours seront célébrés après 1945 comme le lieu de naissance de l'ethnologie française. Doter les futurs observateurs de la vie sociale des «populations indigènes » des colonies de «cadres de classement», tout

en les rendant sensibles au «complexe » et au «concret», à l'intrication de tous les phénomènes, telle était la visée de Mauss. Si l'enseignant avait ses objets de prédilection et ses marottes méthodologiques, son cours n'en apparaissait pas moins comme «une suite de sketchs, un éblouissement » (p. 390), nourri d'exemples prosaïques et personnels, ponctué de courts-circuits rhétoriques ("l'auditeur s'étonnait d'entendre Mauss commencer sa phrase par "Douzièmement": quels étaient les onze points précédents? », p. 394) ou d'observations déconcertantes: «Il faut si possible dresser un indigène à la recherche. C'est ainsi que l'on obtient les meilleurs documents. [...] Un indigène sociologue risque d'arriver au maximum d'exactitude. $)$ (p. 361) Ce portrait de Mauss en professeur éclaire admirablement la «tension » qui fait «le ressort de son activité pédagogique » : le cours concilie «une exigence scientifique totale [...] avec une dimension prosaïque, concrète, terre à terre parfois - un peu transgressive aussi » (p. 399), qui cristallise peut-être en lui l'esprit des temps.

La suite logique de la formation auprès de Mauss est bien sûr le départ en mission «épreuve du feu des futurs maîtres de l'ethnologie », dont C. Laurière expose les principes et les modalités. Au cours des quinze premières années de l'Institut d'ethnologie, plus de soixante-dix missions ethnographiques sont réalisées, dont Mauss, Rivet et GHR assurent le montage et les arrières. L'importance de ces missions témoigne de la puissance de rayonnement de l'ethnologie, comme de son pouvoir d'attraction existentiel et intellectuel; c'est aussi ce que révèle la diversité des provenances sociales, géographiques et universitaires des ethnographes, reporters, documentaristes assurant ces missions, encore généralement collectives, et menées le plus souvent dans les colonies françaises. II n'y a toutefois pas, note C. Laurière, d'«école d'ethnographie maussienne »: «les méthodes d'enquête et leur bricolage in situ, le rapport aux informateurs, les conditions du séjour la construction des objets, les stratégies d'écriture, la reconstitution du savoir engrang varient considérablement d'un ethnologue à l'autre » (p. 431). E† si le MET, puis le musée de l'Homme sont, selon Rivet, un « véritable laboratoire du Colonial » (p. 444), les ethnologues sont, en réalité, assez éloignés des problèmes de gouvernance des populations indigènes: Rivet et Mauss étan plus préoccupés par la position académique de l'ethnologie et sa construction comme discipline, la coopération avec l'administration coloniale est finalement surtout d'ordre logistique.
La collecte est alors le maître mot de ces missions. Mais l'objectif des «collectes, collecteurs, collections », comme le rappelle A. Delpuech, est non pas de rassembler des objets d'art, mais de constituer tout objet en objet de savoir, afin d'y voir un document qui soit «preuve du fait social». Corollaire de la mission de sauvetage dont l'ethnographie s'estime chargée, l'impératif de la collecte donne lieu à une «course contre la montre» et à une «frénésie concurrentielle», les méthodes d'acquisition, plus ou moins scientifiques, relevant parfois clairement de l'extorsion - Griaule dispose ainsi d'un «permis de capture scientifique », délivré par le ministère des Colonies (p. 479). Si l'idée émerge qu'il faut lutter contre le préjugé de l'objet «pur» et collecter des objets hybrides, métissés d'apports occidentaux, ce principe est en fait encore peu mis en pratique dans les années 1930, en raison de la «nostalgie du néolithique », selon le mot de Métraux, qui fait concevoir l'ethnologie comme une «archéologie par anticipation ${ }^{3}$ ».

Les contributions de C. Peltier-Caroff et de D. Villar offrent ensuite deux riches portraits d'américanistes atypiques, Paul Coze et Jehan Vellard. L'examen du parcours et des activités de Paul Coze, indianophile passionné et «peintre des Peaux-Rouges», permet de révéler les liens entre indianisme et scoutisme, qui contribuent à la diffusion de la culture nord-amérindienne en France. Les années 1920 sont en effet marquées par le «peaurougisme », soit la promotion de pratiques et d'activités inspirées par la vie des NordAmérindiens (veillées, jeux de piste, écritures secrètes...), avant que ne leur soit préféré le chevalier, qui cadre mieux avec les valeurs catholiques. Fondateur de Wakanda, Cercle d'art et d'études peaux-rouges, Paul Coze est aussi à l'origine de missions financées par le MNHN et les Scouts de France, de l'exposition «Peaux-Rouges d'hier et d'aujourd'hui », en 1931, ou de l'exposition d'artistes amérindiens contemporains, en 1935. L'étude consacrée à ce personnage singulier éclaire admirablement le continuum entre curiosité vraie, pratiques de détournement culturel et entreprise de «promotion», ainsi qu'entre ethnographie et spectacle.

D. Villar brosse un beau portrait «en clairobscur» de Jehan Vellard, «ancêtre impossible pour l'américanisme » (p. 578), qui a laissé derrière lui «un legs trop encombrant » pour être revendiqué. La restitution de ses expéditions réalisées pour le MET dans les années 1930, en particulier au Paraguay (dans le Chaco, de fortin en fortin, puis en quête des Guayaki) et au Brésil (dans le Mato Grosso, le long de la ligne télégraphique, avec les Lévi-Strauss), renseigne sur les conditions de constitution des collections du musée. Un épisode de ses expéditions auprès des Guayaki retient tout particulièrement l'attention: au terme de l'une d'elles, Vellard adopte une fillette capturée (en même temps que les objets du campement assailli), qu'il prénomme Marie-Yvonne et confie à sa mère (qui l'accompagne dans ses voyages); cette fillette puis d'autres enfants «adoptés » deviennent pour lui des objets d'étude. L'expérience

fascine Rivet, qui voit dans le geste un acte d'humanité, et dans la capacité d'adaptation de la fillette une preuve de l'égalité des races, tandis que d'autres (Curt Nimuendajú et ses disciples) l'envisagent plutôt comme un scandale, «typique de l'insensibilité coloniale » (p. 555). L'intérêt du portrait est de ne pas réduire l'étrangeté du personnage, naturaliste passionné d'anthropométrie, homme d'institutions, indéfectiblement fidèle à Rivet, catholique, mais positiviste et manifestement peu enclin à l'empathie, qui finira par déshériter sa fille adoptive.

Les articles de C. Benoît et A. Delpuech, d'une part, de N. Dias, d'autre part, détaillent deux projets inaboutis ou avortés, parfois plus révélateurs que ceux qui réussirent: 


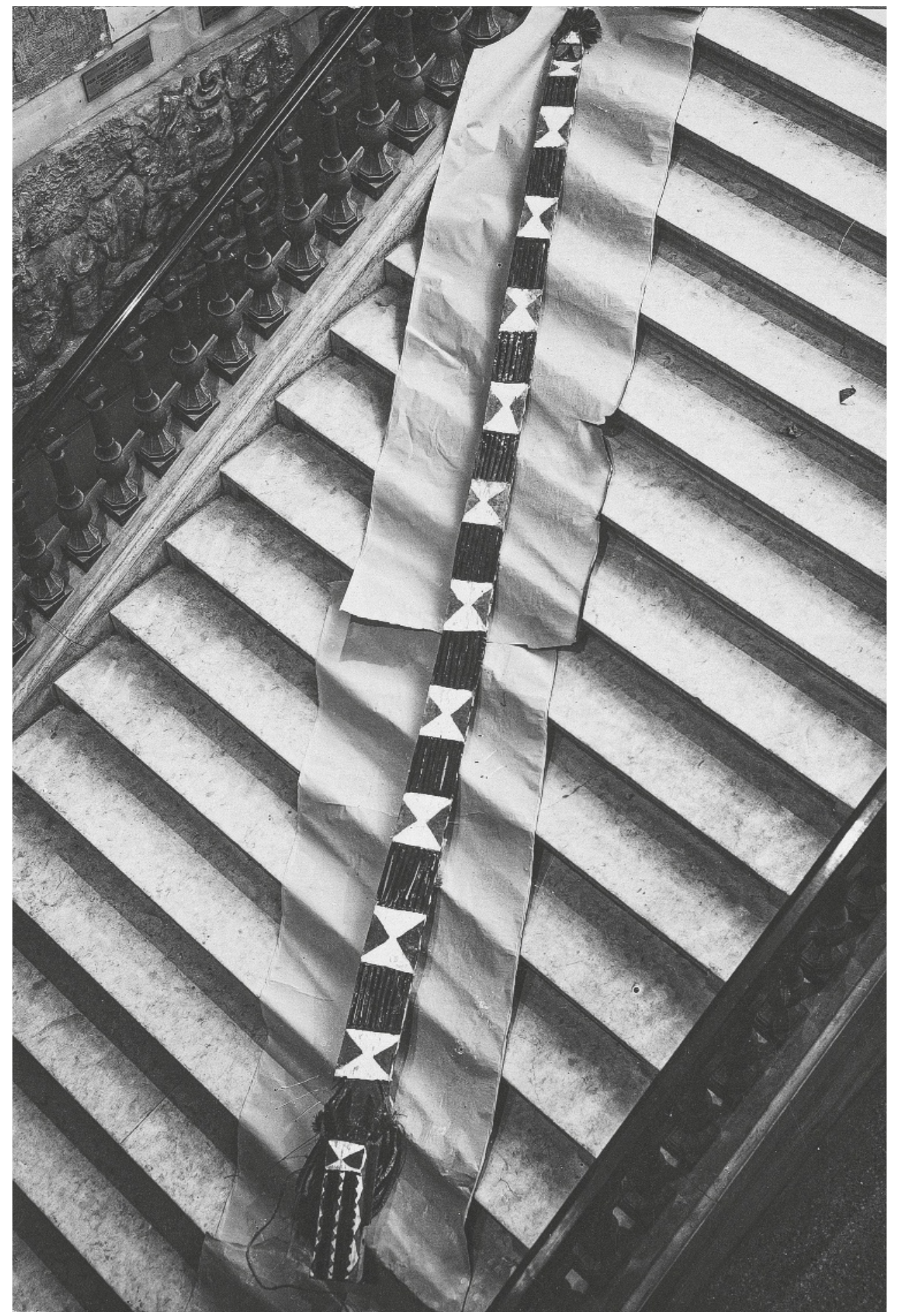

Anonyme: Masque dogon photographié en surplomb, en raison de sa lonǵueur, du haut des marches d'un grand escalier pour obtenir le recul nécessaire à la prise de vue, au musée d'Ethnographie du Trocadéro, 1931. Tirage sur papier baryté. Masque dogon collecté par Henri Labouret au Mali (71.1930.31.21), 5,20 mètres. 

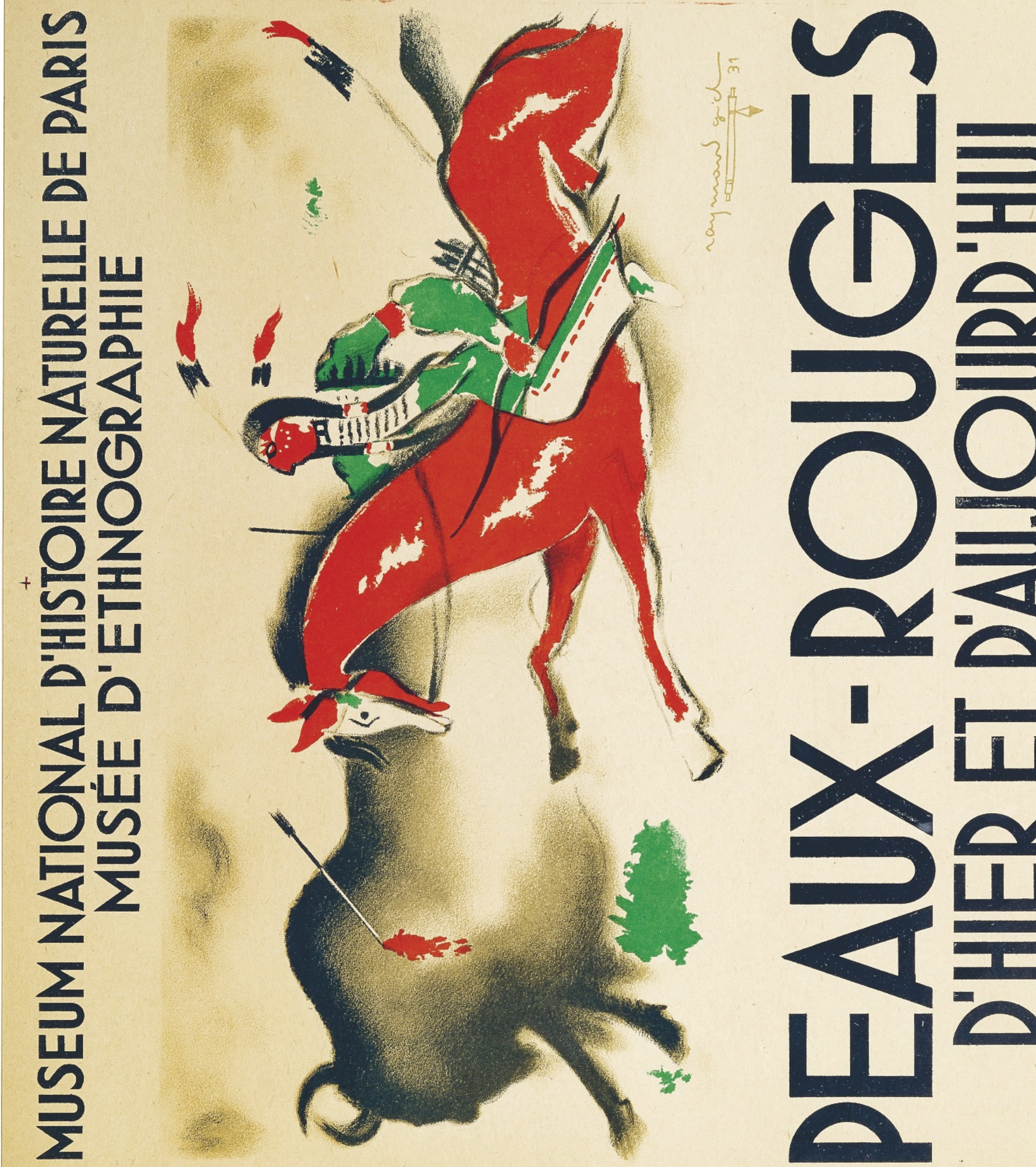


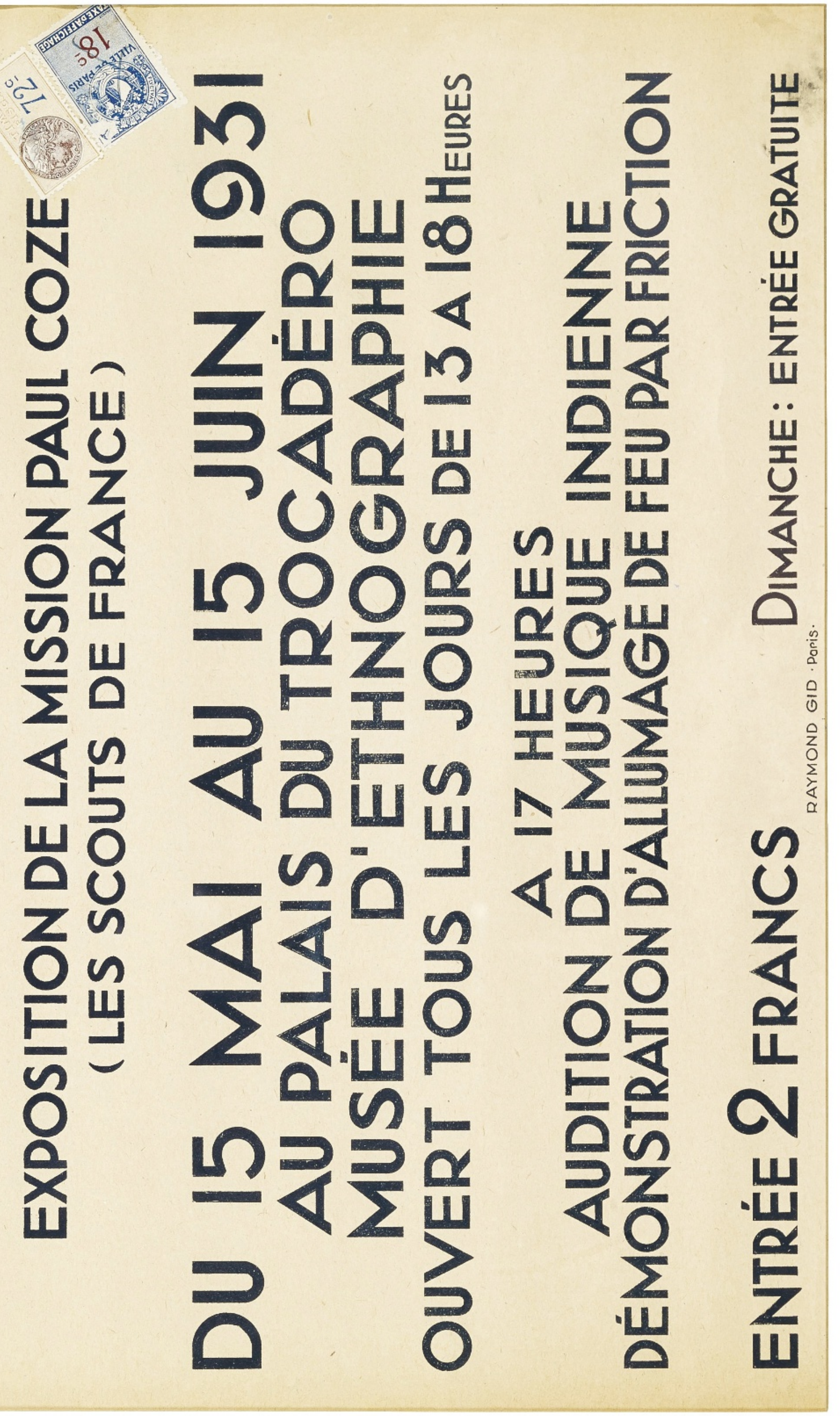




\section{Note de lecture}

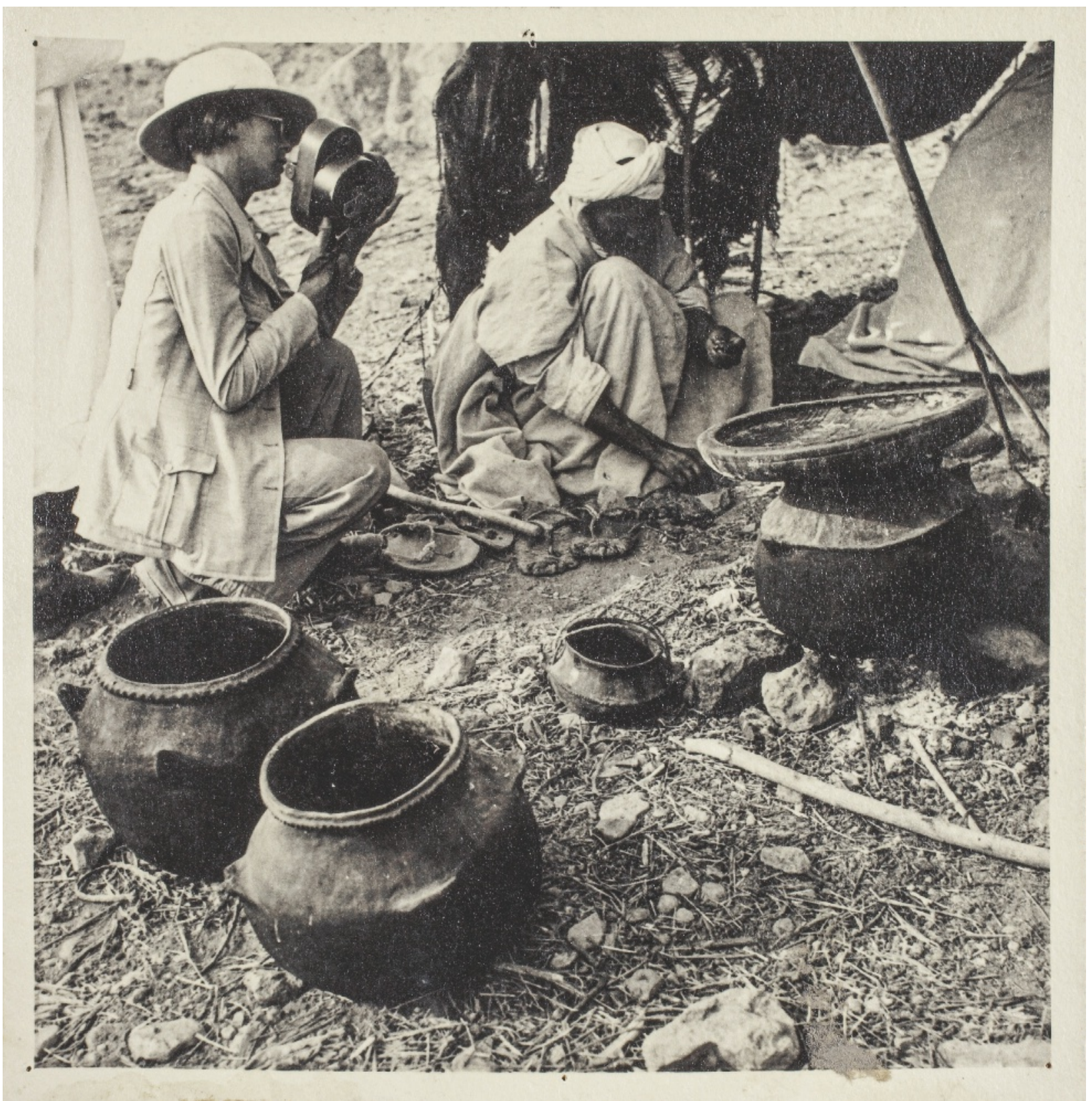

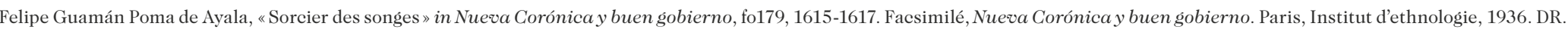

le «rendez-vous manqué avec les "vieilles colonies" "), la Guyane et les Antilles françaises, et les tentatives de promotion de l'ethnologie en Indochine à travers la création d'un musé ethnographique à Dalat. C. Benoît et A. Delpuech restituent le «bouillonnement anthropologique suscité par la région Caraïbe » (p. 581) pendant la décennie, qui apparaît pourtant rétrospectivement comme un faux départ. Ce «bouillonnement» se manifeste par de nombreuses missions (notamment la mission Léon-Gontran Damas, dont les conditions restent à élucider), les investigations archéologiques pionnières dans la région, ou la commémoration du tricentenaire du rattachement des Antilles et de la Guyane à la France, en 1935 . Ce sont là les «prémisses [sic] d'une anthropologie caraïbéaniste globale» (p.618), et non plus centrée seulement sur les communautés autochtones «pures », occultant les réalités coloniales; elles donnent lieu au projet d'exposition «L'Afrique dans les Amériques», en 1934, manifeste pour une ethnologie du monde créole. Ce projet ne verra cependant pas le jour, à cause du conflit mondial, puis de la départementalisation de ces territoires, en 1946, qui tend à les exclure de la recherche pour les chercheurs métropolitains aussi bien que locaux.
L'article de N. Dias explique le projet de creation d'un musée ethnographique à Dalat, antenne du MET, et la mission Rivet en Indochine française (1932), œuvrant à la mise en place d'un réseau pyramida d'enquête et de travail entre colonie e métropole. Cette organisation donne lieu à «un véritable échange d'informations, une circulation d'idées, d'objets et de personnes entre le musée métropolitain et le réseau local situé en Indochine française » (p.659). Le projet d'un musée ethnographique à Dalat soutenu par Rivet, n'aboutit finalement pas à Hanoï, un musée de l'Homme voit cependan le jour en 1938, qui, porté moins par les idéaux de Rivet que par l'idéologie de Vichy, met plutôt l'accent sur les spécificités ethniques del'Indochine. Commele note N. Dias, «peut être ne suffisait-il pas uniquement d'importer en Indochine française des modèles

muséologiques et des pratiques de collecte, de collection; encore eût-il fallu aussi prendre en compte le rôle qu'un musée ethnographique était appelé à jouer dans les formes de gouvernementalité locale » (p. 663)

Enfn, F. Dalex brosse le portrait d'une « exploratrice ethnographique modèle », Madeleine Colani, d'abord institutrice, puis géologue, archéologue et préhistorienne, qui, sexagénaire, devient membre correspondant de l'Ecole française d'Extrême-Orient et multiplie les fouilles en Indochine, doublées de collectes ethnographiques pour le MET. Elle forme, plus exactement, un tandem logistique avec sa sœur Éléonore, qui est peut-être celle qui se charge véritablement du travail ethnographique. Les objets collectés, accompagnés de photographies, qui documentent leur contexte social et leur utilisation, contribuent prodigieusement à l'enrichissement des collections ethnographiques asiatiques du musée (les arts d'Asie étant jusqu'alors massivement conservés au musée Guimet et dans les musées de province); réceptionnés au MET par Marcelle Bouteiller, ces objets sont montrés, notamment, lors de l'exposition sur les « Jouets annamites », en décembre 1932, qui se solde par une vente aux enchères.

Cette recherche sur les sœurs Colani met en valeur le travail de deux actrices clés, quoique méconnues, de l'ethnographie indochinoise.

Le troisième volet, «Médiatisation de l'ethnographie. Photographies, expositions, radio et livres "), s'attache à la production iconographique, médiatique ou livresque qui accompagne l'ethnologie, au-delà de l'exposition d'objets. 
Dans cette configuration élargie du savoir, la photographie joue un rôle considérable. A. Mauuarin analyse ainsi les logiques de constitution des collections photographiques au musée, qui va donner lieu à l'organisation d'une photothèque L'intérêt pour le médium, dans le fil des projets de documentation mondiale autour de 1900 (telles «Les archives de la planète », d'Albert Kahn), procède du désir d'exhaustivité et du sentiment d'urgence qui accompagnent l'ethnologie, en même temps qu'il constitue un moyen de renforcer l'autorité scientifique de la discipline.

Dès 1928, un service spécifique s'organise, et l'usage du terme «photothèque» se stabilise en 1933; Thérèse Rivière assure le fonctionnement du service et l'organisation des documents; un photographe entre en poste au Trocadéro à partir de 1933, et un laboratoire est mis en place au sein du musée. Il faut distinguer, au sein des collections, les photographies prises par les voyageurs et les ethnologues, d'une part, et les photographies d'objets conservés au musée et de vues de la muséographie, d'autre part. Ces dernières participent du processus de rationalisation et de valorisation des collections; le «portrait» photographique de l'objet tend en outre à lui faire changer de statut, de l'artefact à l'objet d'art (Charles Ratton fait ainsi photographier sa collection par Walker Evans). Dans les expositions d'objets ethnographiques, la photographie a souvent une vocation de médiation entre collections et public: elle permet de montre les objetsen usage, les vêtements portés, etc.; mais le musée organise également des expositions de photographies: on en compte dix entre 1932 et 1935 . C'est l'indice d'un changement de statut des photographies: d'abord simples éléments de documentation, à portée pédagogique, elles sont auss des objets à part entière, dotés de qualités esthétiques propres, et attirant un vaste public.

A. Loyaux ouvre pour sa part les coulisses de l'exposition «Sahara », qui, en 1934 a fait du musée «un improbable terrain de coopération des nations coloniales européennes » (p. 760). Si l'ambition initiale de cette exposition était de populariser des savoirs contemporains sur le Sahara, d'autres enjeux interfèrent bientôt, puisque les nations étrangères ayant des possessions dans ce territoire sont invitées à y participer. À travers l'examen des préparatifs et de la muséographie de l'exposition, ainsi que de l'accueil qui lui est réservé, l'étude dévoile ses dimensions coloniales et internationales, mettant au jour les modalités de participation variables des différentes puissances coloniales, et surtout les options divergentes entre la France et I'Italie au milieu des années 1930: Rivet soutient un projetencyclopédique universaliste, quand I'Italie fasciste, par ses choix, soutient son propre projet de société, choix, soutient son propre projet de société, la rhétorique d'«union sacrée coloniale» déployée à l'occasion vise avant tout à légitimer le processus colonial français et à promouvoir une image généreuse de ses propres ambitions (p. 772). Rejouant un peu, par son ampleur, I'Exposition coloniale de 1931, I'exposition «Sahara » incarne ains la collusion entre science ethnologique, entreprise coloniale et jeux diplomatiques.

L'ethnologie a aussi su inventer sa place sur les ondes. La contribution de M. Lemaire analyse un cycle de radio-conférences mis en place par le MET, données de 1935 à 1939, soit un corpus de cent vingt-six conférences (des textes dactylographiés, sans archives sonores). Conçu en 1935 par GHR et Pierre Bertaux, responsable des émissions parlées de Paris P.T.T, ce cycle, intitulé «Voyages et explorations », permet d'observer les relations entre voyage, ethnographie et radio dans l'entre-deuxguerres. Les conférenciers sont pour la plupart des ethnologues proches du musée et des naturalistes rattachés au Muséum, mais aussi des «explorateurs», reporters photographes, hommes de lettres, sportifs ou artistes; un quart d'entre eux sont des conférencières, Rivet consacrant lui-même deux interventions (reproduites en annexe) à la contribution des femmes à l'ethnologie. Ces conférencières font d'ailleurs sur les ondes des choix différents de ceux qu'elles opèrent dans leurs publications, s'autorisan alors à discuter de leurs propres expériences de terrain et du sort réservé aux femmes dans les cultures étudiées. Tout comme la presse, la radio-conférence est un moyen de promouvoir la recherche ethnologique et de gagner la curiosité du plus grand nombre: ces conférences représentent une invitation au voyage, le plus souvent en territoires lointains, parfois en France. Les intervenants, exprimant quelquefois le regret de ne pas pouvoir montrer de photographies ou films, s'essayen à restituer l'atmosphère sonore des région évoquées, faisant alors preuve d'inventivité dans l'emploi du médium: le recours

à des enregistrements ou des citations de littérature indigène, I'invention de formes dialoguées, etc. Portées par un projet idéologique et politique comparable, radio et ethnologie ont, dans l'entre-deux-guerres, des visées convergentes: offrir un accès égalitaire à la culture, et faire sentir à l'auditoire la «commune humanité » entre peuples.

En clôture, V. Debaene retrace le destin de a collection «L'Espèce humaine», des édition Gallimard. Imaginée dès 1935, et initialemen conçue comme une production collective

du MET, elle sera finalement absorbée, après un régime de parution variable, dans la «Bibliothèque des sciences humaines », en 1965. À partir de la correspondance d'Alfred Métraux, conseiller scientifique de la collection auprès de Brice Parain jusqu'e 1939 , V. Debaene reconstitue la gestation et les déboires de la collection, révélateurs des possibles de l'ethnologie dans les années 1930. L'analyse éclaire les «trois "scènes" sur lesquelles l'invention de cette collection prend place » : «la rivalité entre ethnologie et géographie humaine »,

la collection contribuant à l'autonomisation de la discipline ethnologique; «l'inscription de l'ethnologie française dans un paysage de l'ethnologie française dans un pays disciplinaire international, marqué en
particulier par l'essor de l'anthropologie américaine», que Métraux a à cœur de rendre accessible au public français «la définition de la discipline dans ses rapports avec la production grand public et littéraire (p. 834). Ces derniers obéissent à deux (p. 834). Ces derniers obéissent à deux
tendances distinctes: la nostalgie des belleslettres (style Mauss), et le désir de ressuscite "la vie même » par la grâce du document (style Bataille). Ces deux tendances guident Métraux dans l'écriture de son Île de Pâques, qui paraîtra dans la collection sous deux versions (la seconde expurgée, délestée du «vécu»); il ressortira finalement insatisfait et amer de ces diverses collaborations. Au final, la collection «L'Espèce humaine » apparaît comme «un lieu imaginaire, un espace pour une communauté rêvée », incarnant l'esprit du Trocadéro, à travers «le projet d'une ethnologie lisible et accessible à tous $\gg($ p. 870-871). La répartition de ses ambitions, dans les années 1960, entre la «Bibliothèque des sciences humaines 》 et «Terre humaine » est elle-même révélatrice du changement de «modèle de relations entre ethnologie et littérature survenu après-guerre.

Au terme de ce parcours, le MET, cœur de l'ethnologie française de l'entre-deuxguerres, se révèle comme une machinerie complexe, à l'activité à la fois trépidante et bien réglée, microcosme et projet à l'échelle de la planète. Tout l'intérêt du volume est de mettre en lumière la diversité des pratiques épistémiques rattachées au musée, et l'unite du projet intellectuel et politique qui les porte, dans des études qui prennent parfois pour objet un fait en apparence mineur, une figure méconnue, des initiatives variablement abouties. On peut s'interroger, en définitive, sur la singularité de ce moment culturel. La «folie» de ces Années folles, ce son d'abord l'enthousiasme et l'esprit de subversion qui y règnent, ceux d'un temps où un combat de boxe peut financer une mission ethnographique, et un vénérable professeur prendre la pilosité de ses propres mollets comme exemple dans un cours traitan des traits distinctifs des races humaines (p. 391); c'est aussi celle d'un temps où l'entreprise coloniale est encore peu questionnée, où le succès des expéditions se mesure d'abord à la quantité des objets recueillis, et où il est possible à un Vellard d'écrire, après une fusillade dans un campement guayaki: «Ne pouvant nous charger du cadavre, je me contentai de le mesurer » (p. 573). Ces folles années sont aussi des années de normalisation scientifique des formes du «goût de l'autre », de rationalisation des procédés de constitution et de gestion des collections, et de grande maîtrise des procédés de communication, où débrouillardise inventive et pensée stratégique agissent en synergie.

Si les différentes contributions se font écho et s'éclairent mutuellement, une alternative méthodologique, qui aurait l'avantage de tracer nettement des coupes transversales entre les diverses histoires restituées, serait d'écrire la «biographie » d'objets: suivre la vie d'un objet donné, en considérant ce que chacun des acteurs impliqués (producteur, collecteur, conservateur, commissaire, visiteur d'exposition) en fait successivement, révélerait ainsi les diverses cosmographies dans lesquelles il transite et prend sens ${ }^{4}$. Mais, comme le notait Mauss, «une petite tribu est un monde, pour la décrire, il faut plusieurs volumes: rappelons-nous cette vérité » (p. 392), et nul doute que ces propos valent aussi pour l'historien des savoirs.

Éléonore Devevey eleonore.devevey@gmail.com
C'est ce à quoi invite, en clôture de sa contribution, A. Martin, renvoyant notamment à l'article d'A. Delpuech,

«Histoires de capes et d'epées.

Objets tupinamba entre Brésil et France», in Sabine Du Crest (dir.)

Exogénèses: objets frontière

dans l'art européen (XVI ${ }^{e}-X X^{e}$ siècle). Paris, Boceard, 2017: 39-55. 